\title{
Evaluating intentional quality rounding for undergraduate student nurse training during COVID-19
}

\author{
Shea Polancich ${ }^{* 1,2}$, Connie White-Williams ${ }^{2}$, Laura Steadmann ${ }^{1}$, Kaitrin Parris ${ }^{1}$, Gwen Childs ${ }^{1}$, Terri Poe ${ }^{2}$, Linda \\ Moneyham ${ }^{1}$ \\ ${ }^{1}$ UAB School of Nursing, University of Alabama at Birmingham, Birmingham, AL, United States \\ ${ }^{2}$ UAB Hospital, University of Alabama at Birmingham Hospital, Birmingham, AL, United States
}

Received: September 21, 2021

DOI: $10.5430 /$ jnep.v12n5p47
Accepted: December 7, 2021

Online Published: January 5, 2022

URL: https://doi.org/10.5430/jnep.v12n5p47

\begin{abstract}
Nursing's body of knowledge is ever expanding, incorporating new theoretical constructs such as quality and safety and care transitions we now consider central to the domain of nursing, and to nursing clinical education. The purpose of this article is to describe an educational quality improvement project, an alternative clinical learning experience during COVID-19 that enabled the implementation and evaluation of Bachelor of Science in Nursing (BSN) students in an intentional quality rounding process. We designed and implemented a retrospective, observational quality improvement educational project. Programmatic evaluation was used to obtain feedback from 273 pre-licensure students using a 10-item Likert scale evaluation tool in June 2020. Students averaged a 4.33 rating on the evaluation of the intentional quality rounding clinical experience as something they should incorporate into future nursing practice. A critical role for nursing education is the development of innovative teaching strategies and learning experiences that facilitate the student in the translation and application of complex constructs from nursing's expanding body of knowledge, a task made more difficult by the COVID-19 pandemic.
\end{abstract}

Key Words: Clinical nursing innovation, COVID-19, Programmatic evaluation, Quality improvement

\section{INTRODUCTION}

The provision of quality care with the goal of a safe patient experience are necessary and expected outcomes for the hospitalized patient. Nursing care is a primary driver for quality and safety in the hospital environment. Successful and competently performed nursing interventions such as the placement of a foley catheter or the management of a central vascular device prevent the hospitalized patient from developing infections, while failure to follow evidencebased protocols for aseptic technique and sterile procedures increase patient morbidity, and potentially mortality. ${ }^{[1-3]}$
Bedside nurses, as well as nurse trainees and nursing students, should possess quality and patient safety competencies that allow them to evaluate the patient care environment, the workflow and processes, in order to avoid latent risk-factors for patient harm. Training programs for preparing nurses are typically focused on the delivery of care, specific to the anatomy and physiology of the patient. ${ }^{[4]}$ While these scientific underpinnings of patient care are necessary elements of nursing education, the safe delivery of evidence-based, quality care may not be fully realized in the training environment. Pre-licensure or undergraduate curriculum development may lack sufficient information and applied clinical hours on

\footnotetext{
*Correspondence: Shea Polancich; Email: spolancich@uabmc.edu; Address: UAB School of Nursing, University of Alabama at Birmingham, Birmingham, AL, United States.
} 
the impact of nursing practice related to patient quality and safety.

Nursing education is often based on maturation in learning on a continuum, such as Benner's ${ }^{[5]}$ "Novice to Expert," like any other student of a discipline. Developing any skill requires experiential learning, the premise behind the nursing student clinical training or experience. Developing nursing student quality and safety competencies and the skills for translating best evidence into practice requires training, education, and clinical exposure.

The 2020 Corona Virus or COVID-19 ${ }^{[6]}$ pandemic resulted in challenges to ensuring comprehensive clinical education for trainees in all health professions, specifically for nursing education. Leaders preparing undergraduate nurses have faced barriers to ensuring adequate clinical time due to COVID-19. Comprehensive training for the student nurse requires a combination of clinical, laboratory, and didactic education. ${ }^{[4]}$ For the pre-licensure student, lack of adequate clinical time does not allow for the development of fundamental nursing skills, nor the opportunity for the acquisition and translation of quality and safety awareness associated with quality nursing care.

Nursing's body of knowledge is ever expanding, incorporating new theoretical constructs such as quality and safety and care transitions we now consider central to the domain of nursing. A critical role for nursing education is the development of innovative teaching strategies and learning experiences that facilitate the student in the translation and application of such complex constructs in a meaningful way so as to improve the outcomes of nursing practice. Clinical learning experiences are critical to student understanding of the complex constructs associated with quality and patient safety, and their application in the provision of nursing care. $^{[7,8]}$

\subsection{Aim/Objective}

ThisThe purpose of this article is to describe an educational quality improvement project, an alternative clinical learning experience during COVID-19 that enabled the implementation and evaluation of Bachelor of Science in Nursing (BSN) students in an intentional quality rounding process. The evaluation process was two-fold. The first goal was to assess the clinical experience, and the second goal was to determine the effectiveness of the intentional quality rounding approach for raising awareness of quality and safety aspects of care delivery.

\subsection{Background}

The School of Nursing (SON), a focus of this project, like many other educational programs, has been faced with iden- tifying alternative clinical learning experiences that are safe and also support students in meeting required clinical hours so that they can continue to progress through a program of study and graduate. Student clinical learning experiences were suspended in mid-March 2020 by restrictions imposed by the university as the cases of COVID-19 began to increase in the state. The state Board of Nursing also allowed student progression within a curriculum if 50\% of the required clinical hours were completed and the students had met course objectives. As cases began to decline and hospitals began to return to normal operations, faculty began to plan for return of students to clinical settings in summer semester 2020 , working within the limitations of the clinical learning environment due to COVID-19.

The leadership of the SON and its primary clinical partner hospital met to determine if there was an opportunity to create some type of clinical learning experience that would allow pre-licensure, clinical nursing students to return to the clinical environment and accrue hours in direct patient care once approvals for re-entry were obtained by the university and the hospital. The student learning experience also needed to conform to limitations in direct patient care hours, and subsequent limitations in the skills the students would be able to perform with the restrictions imposed.

More than 400 pre-licensure students were in need of clinical experiences in direct patient care, and priority for placement was determined based on length of time until graduation, with students in their last two semesters given priority for direct patient care experiences. For the remaining students, who were in their second and third semesters of the BSN program $(n=275)$, there were fewer opportunities for oneto-one direct patient care experience. Faculty had developed numerous virtual clinical learning experiences for the second and third year students to support their continued development in key areas appropriate to their level such as health assessment and other psychomotor skills including medication administration, and urinary catheter placement to name a few, as well as critical thinking and problem solving through high-fidelity simulation and case study methods. The faculty goal for these students was to secure at least limited clinical experiences with direct patient contact that would support their on-going development and application of critical curriculum concepts, and build on the development of nursing awareness of safe, quality care delivery.

\section{Methods}

Clinical nursing students were approved by the university to return to the clinical environment as of June 1, 2020. Leadership from the SON and the partner hospital identified a novel clinical experience for the 2nd and 3rd semester stu- 
dents that allowed them the opportunity to participate in scheduled intentional quality rounding events, and would facilitate each student obtaining 18 clinical hours or $20 \%$ of the total required clinical hours in the hospital. The authors implemented and evaluated a retrospective, observational, educational quality improvement (QI) project using the intentional rounding process.

The novel element of the QI clinical experience was the introduction of intentional quality rounding. While students are often instructed on elements associated with quality and patient safety, such as bedside handovers/handoffs, ${ }^{[9]}$ the intentional quality rounding is focused on evidence based best practices (EBP) for reducing adverse patient outcomes. Intentional quality rounding EBP elements often include focus areas for pressure injuries, patient falls, infections, and restraint use. ${ }^{[1]}$

Students were integrated into the quality rounding teams with the role of performing a quality assessment of all patients on a unit for the identified adverse patient outcomes. Carried out on numerous patient care units on the same day, the rounding strategy provides the hospital with metrics for ongoing evaluation of the effectiveness of interventions to reduce adverse outcomes. For students, intentional rounding provided them the opportunity to participate in real-time application of quality and safety principles as applied at the individual and health system level. Evidence-based practice, quality and patient safety are major constructs in the BSN curriculum that students may not appreciate nor see applied in the clinical practice setting. Consequently, the intentional rounding experience provided the opportunity to evaluate its impact on students understanding of the nurse's role in evidence-based practice and quality improvement.

The students were trained on the protocol for intentional quality rounding, including steps for assessment of each of the adverse outcomes to be assessed that day as well as the evidence-based protocols in place. Students were expected to provide a quality assessment for each patient encountered. Hospital nursing staff and graduate nursing students were available to provide oversight and resources for the students. Following the initial patient quality assessment, student were asked to make rounds on the patients hourly for ongoing assessment of any change in patient outcomes protocol implementation using quality rounding methods and a checklist. Nursing students may use checklists to facilitate memory for performing tasks or common nursing procedures. ${ }^{[10]}$

\subsection{Program evaluation}

The authors utilized a program evaluation process to evaluate the clinical experience. Program evaluation is defined as a systematic method for collecting, analyzing, and using information to assess effectiveness and efficiency of a project or program, in this case a pre-licensure clinical nursing experience. ${ }^{[11]}$ Summative evaluation was used in the form of an end-of-clinical paper-based questionnaire, also referred to as the evaluation tool.

\subsection{Setting}

The setting for this project is an urban academic health science center located in the southeastern United States where the academic partner is a School of Nursing and the clinical partner is the Academic Health Center (AHC). The academic partner is a Commission on Collegiate Nursing Education(American Association of Colleges of Nursing ${ }^{[12]}$ accredited degree granting school of nursing offering Bachelors, Masters, and Doctoral level nursing degrees. The SON enrolls on average approximately 2,500 students per year and matriculates an average of 1,000 students annually in the combined programs (based on 2018-2019 data). The SON, as part of a renowned AHC, received more than 12 million dollars in extramural funding in 2019 and is consistently ranked among the top ten public nursing schools for its academic and research programs. ${ }^{[13]}$

The AHC is the 3rd largest public hospital in the United States. The medical center has 1,207 beds with an average of 55,000 admissions per year and 6,000 ambulatory visits per day. The AHC also employs 1,400 physicians, 3,600 nurses, 800 advanced practice providers, and since 2002 has received ANCC Magnet designation ${ }^{[14]}$ in nursing five consecutive times $(2002,2006,2011,2015,2019)$, making it one of an elite number of organizations world-wide to receive this number of designations.

\subsection{Institutional review board}

Institutional Review Board approval was obtained for conducting the evaluation of the novel clinical experience under the auspices of quality improvement and non-human subject (Federalwide Assurance Number FWA00005960, IORG Registration \#IRB00000196 (IRB 01), IORG Registration \# IRB00000726 (IRB 02)). Students were not required to participate in the clinical activity given the pandemic, and alternative clinical experiences were created for those uncomfortable with return to the hospital. The evaluation tool used in the project was voluntary and anonymous, and student participation in the feedback implied consent as stated in evaluation instructions.

\subsection{Design of the pre-licensure intentional quality rounding clinical experience}

Leaders from the hospital and SON designed the intentional quality rounding process to include assessment of patients 
for the implementation of evidence-based best practice guide- tient assessment at the beginning of a nursing shift, then lines for four specific quality focus areas: reducing falls and pressure injury, restraint management, and infection prevention for urinary catheter and central line placements. The optimal intentional quality round begins with an initial pahourly rounding on the patient during the shift to ensure that elements of best practice related to the quality focus areas described were appropriately implemented. Figure 1 provides an overview of the quality rounding experience.
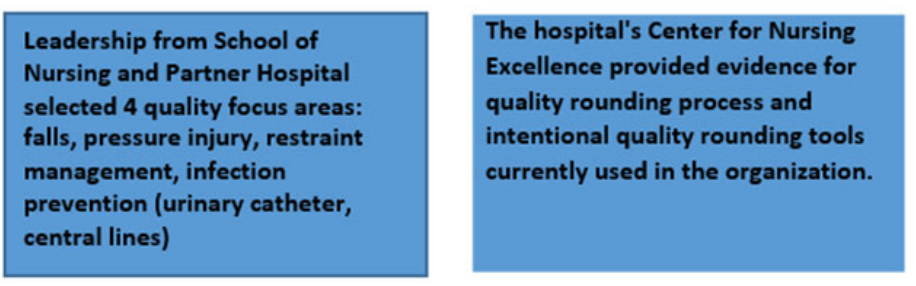

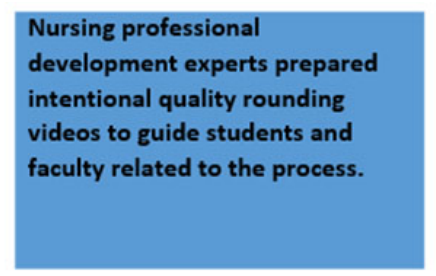

\section{Quality Rounding Development (3 weeks-May 2020)}

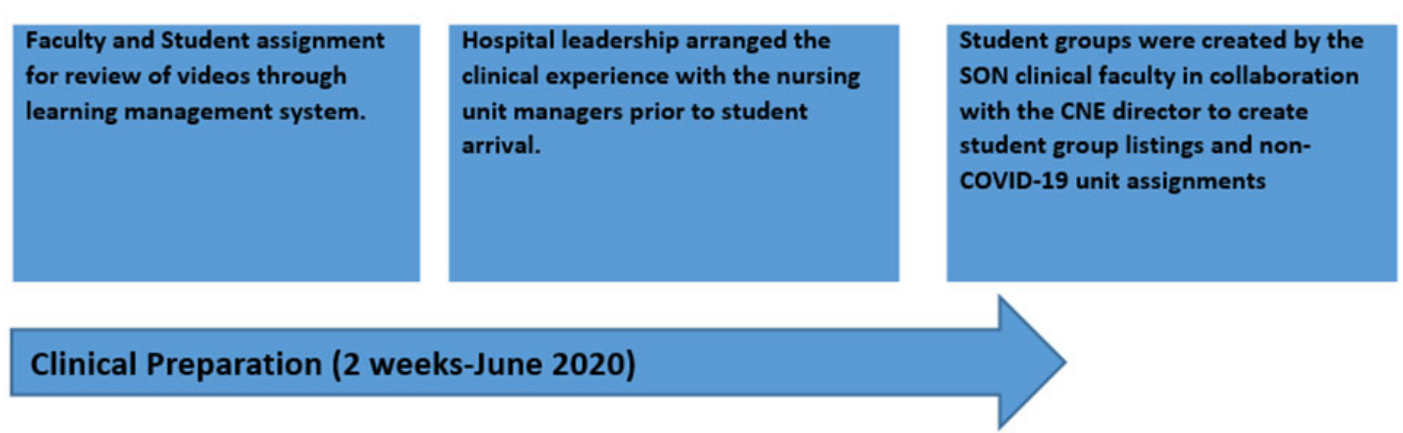

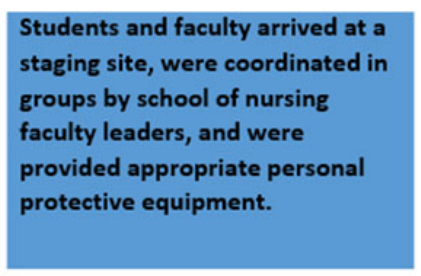

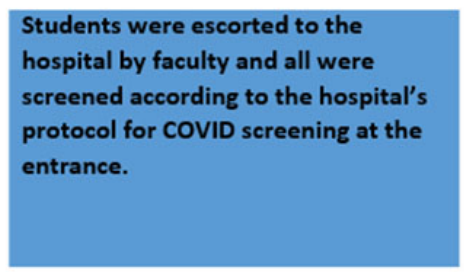

Implementation (3 days-June 2020)

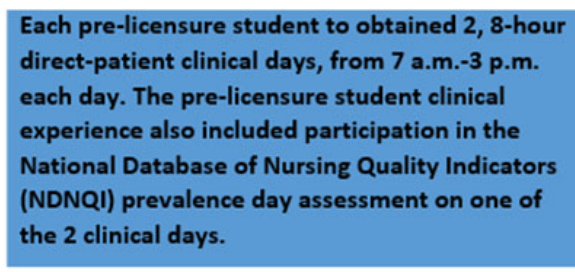

the 2 clinical days.

Figure 1. Design of the pre-licensure intentional quality rounding clinical experience

Prior to the clinical experience, students were provided with a series of videos specific to the intentional quality rounding process, accounting for 2 hours of clinical time. The hospital's Center for Nursing Excellence (CNE) staff organized the content for the intentional quality rounding process using nurse experts from the medical center specific to the quality focus areas. On the day of the clinical experience, the students were provided a rounding checklist aligned with the quality focus areas. For each of the quality focus areas, 3-5 evidence-based practice elements are listed. The checklist is arranged according to hours during a nursing shift. Figure 2 provides an example of the intentional quality rounding checklist.

The hospital's CNE staff arranged the clinical experience with the nursing units. The Senior Director of the CNE communicated with nursing unit managers across the organization to determine willingness to participate in the clinical experience. Student groups were created by the SON clinical faculty in collaboration with the CNE director to create student group listings and non-COVID-19 unit assignments. The student groups and unit assignments were developed to provide as much consistency for the students, allowing them to complete their hours on one unit. 


\section{Patient Room Number}

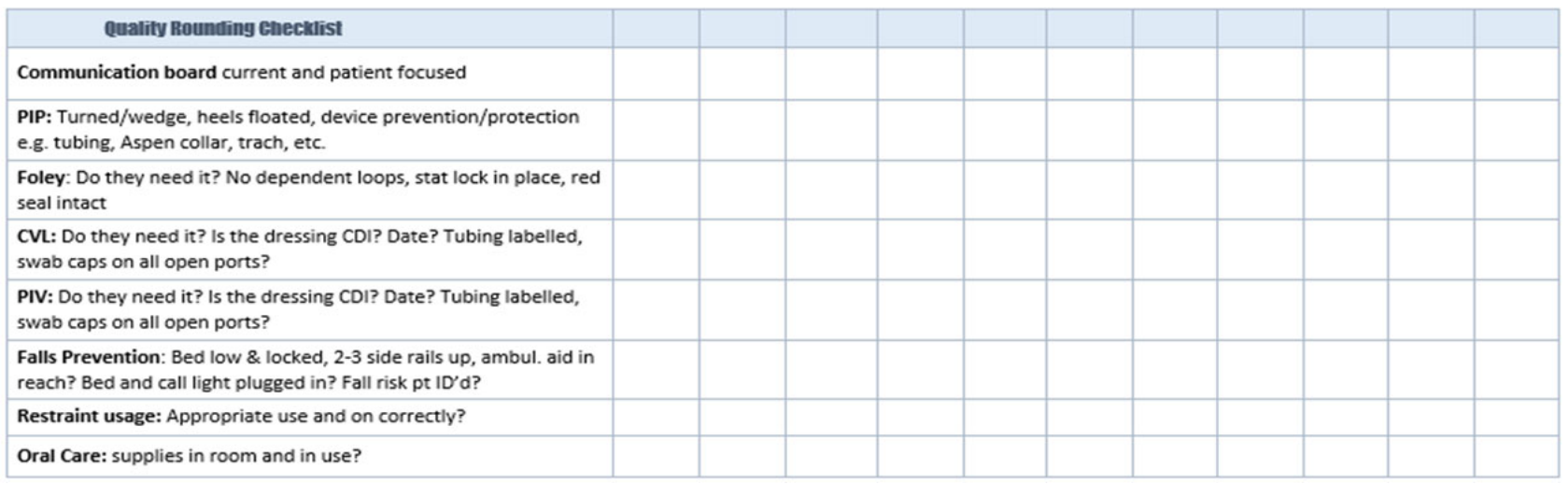

Figure 2. Intentional quality rounding checklist

Legend: PIP-Pressure Injury Prevention, CVL-Central Venous Line, PIV-Peripheral Intravenous Line/Catheter

The hospital team developed and implemented a formal COVID-19 screening process for the clinical experience. All pre-licensure students, Nurse Educator students, and faculty were required to complete COVID-19 symptom screening prior to entry into the clinical environment on the day of the experience. Hospital staff conducted the screening which included both a temperature screen and a COVID-19 symptom checklist, at the hospital entrance.

The clinical experience was designed to be completed in 3 days, ensuring that all 2ndand 3rd semester students were able to obtain the required number of clinical hours. Each pre-licensure student obtained 2, 8-hour direct-patient clinical days, from 7 a.m.-3 p.m. each day for a total of 16 hours. When combined with the 2 hours of indirect clinical hours obtained from the intentional quality rounding video review, a total of 18 hours of clinical time was established. Half of the students were assigned to a clinical experience on day 1 , while the other half were assigned on the 2 nd day. All students were assigned clinical groups on hospital units on the 3rd day of the experience.

The student clinical experience also included participation in the National Database of Nursing Quality Indicators (ND$\mathrm{NQI})^{[15,16]}$ prevalence day assessment. NDNQI prevalence day is a specific day each quarter when all patients in the organization are evaluated for pressure injury and the use of restraints. These data are collected and submitted to a national database. On the third day of the experience all students participated in the NDNQI prevalence day activities by assisting the nursing staff with observation, turning and dressing changes.

The intentional quality rounding process was performed with groups of approximately 8 pre-licensure students assigned to an oversight $\mathrm{RN}$ or clinical faculty member. SON Nurse
Educator (NE) students were allowed to participate in the process, and to provide additional clinical oversight. The NE students are bachelors or masters prepared nurses who are employed at the medical center, but are also students in the SON Nurse Educator Subspecialty track. These NE students were provided an opportunity to voluntarily participate in the process for a course assignment in the nurse educator program. The NE students requested vacation or paid time off days to participate in the clinical experience. Full-time SON clinical faculty provided primary oversight for the clinical experience, and rounded on the student groups on assigned clinical units.

\subsection{Data collection and analysis}

The authors designed a paper-based evaluation tool in the form of a questionnaire using a 5-point likert scale ${ }^{[17]}$ to evaluate the pre-licensure student clinical experience. The evaluation tool provided an opportunity to analyze the experience both quantitatively and qualitatively, and to specifically address the goals defined. The 5-point likert scale responses include strongly disagree, disagree, neither agree or disagree, agree, and strongly agree. The evaluation tool contains 10 statements to which students were to respond how strongly they agreed with each statement: 1) The patient assessment and intentional quality rounding process provided a good opportunity for me to gain clinical experience; 2) The clinical experience was organized and efficient, 3) The videos provided sufficient training for me to feel comfortable performing the process; 4) The patient assessment and intentional quality rounding process taught me skills that I had not learned or mastered in other semesters; 5) The patient assessment and intentional quality rounding process assisted me to develop a view of patient care from a total quality perspective; 6) In comparison to previous clinical experiences, the patient assessment and intentional quality rounding process 
was enjoyable; 7) The unit that I was on provided a diverse group of patients to evaluate; 8) The oversight provided was appropriate and I felt supported to learn; 9) If possible, I would choose to spend additional clinical hours participating in this process; 10) The patient assessment and intentional quality rounding process is something that I will incorporate into future nursing practice. The tool also included an open-ended item for students to provide comments.

Five of the items on the evaluation tool (items 1, 4, 5, 6, 10) were specifically designed to evaluate nursing student's awareness of the nurses' role in quality and patient safety, one of the project evaluation goals. The remaining 5 questions on the evaluation tool were specific to the process and organization of the clinical experience, the second project evaluation goal. The evaluation tool was provided to the studentfor completion at the end of each clinical day.Students were asked to complete the evaluation form only once. The paper-based tool responses were entered by a program assistant into an excel ${ }^{\mathrm{TM}}$ spreadsheet. ${ }^{[18]}$ The authors analyzed these data with descriptive statistics ${ }^{[19,20]}$ calculating mean scores for each item, using the Statistical Package for the Social Sciences ${ }^{[21]}(\mathrm{SPSS})^{\mathrm{TM}}$ analytical software. The single, open-ended question was evaluated for themes and quantified for a mixed methods analysis.

\section{RESUlTS}

A total of $\mathrm{N}=273$ pre-licensure student responses were collected from the 2 nd and 3rd semester students, reflecting $100 \%$ participation. Mean scores from the evaluation ranged from 3.29-4.33. Students mean responses to Item 10 of the tool, "The patient assessment and intentional quality rounding process is something that I will incorporate into future nursing practice" were the highest among all itmes with a mean of 4.33. The lowest student mean responses were for Item 4 , The patient assessment and intentional quality rounding process taught me skills that I had not learned or mastered in other semesters" with a mean of 3.29. Six of the 10 questions averaged scores were greater than 4.0. Table 1 provides a summary of the mean scores for each question.

Table 1. Pre-licensure student evaluation mean scores

\begin{tabular}{|l|l|l|l|}
\hline Goal 1: Evaluation of the Processes and Organization of the Clinical Experience & Mean Score & $\begin{array}{l}\text { Median } \\
\text { Score }\end{array}$ & $\begin{array}{l}\text { Standard } \\
\text { Deviation }\end{array}$ \\
\hline 2. The clinical experience was organized and efficient. & 3.99 & 4.00 & .994 \\
\hline 3. The videos provided sufficient training for me to feel comfortable performing the process. & 4.13 & 4.00 & .795 \\
\hline 7. The unit that I was on provided a diverse group of patients to evaluate. & 4.22 & 4.00 & .929 \\
\hline 8. The oversight provided was appropriate and I felt supported to learn. & 4.41 & 5.00 & .718 \\
\hline Goal 2: Evaluation of the Student Nurse Awareness of Quality and Patient Safety & Mean Score & & 4.00 \\
\hline $\begin{array}{l}\text { 1. The patient assessment and intentional quality rounding process provided a good } \\
\text { opportunity for me to gain valuable clinical experience. }\end{array}$ & 4.03 & 0.968 \\
\hline $\begin{array}{l}\text { 4. The patient assessment and intentional quality rounding process taught me skills that I had } \\
\text { not learned or mastered in other semesters. }\end{array}$ & 3.29 & 3.00 & 1.286 \\
\hline $\begin{array}{l}\text { 5. In comparison to previous clinical experiences, the patient assessment and intentional } \\
\text { quality rounding process was enjoyable. }\end{array}$ & 4.05 & 4.00 & .850 \\
\hline $\begin{array}{l}\text { 6. The patient assessment and intentional quality rounding process assisted me to develop a } \\
\text { view of patient care from a total quality perspective. }\end{array}$ & 3.55 & 4.00 & 1.197 \\
\hline 9. If possible, I would choose to spend additional clinical hours participating in this process. & 3.40 & 4.00 & 1.317 \\
\hline $\begin{array}{l}\text { 10. The patient assessment and intentional quality rounding process is something that I will } \\
\text { incorporate into future nursing practice. }\end{array}$ & 4.33 & 4.00 & .743 \\
\hline
\end{tabular}

Themes were identified from the single open-ended item on the evaluation tool. There were 65 comments entered by the 273 students (24\%). Student responses were categorized into three major themes: organization of the process, value of the experience, and skill development. Some of thestudents commented that the organization of the process could be improved 5/65 (8\%), and communication regarding the experience could be better $5 / 65(8 \%)$. Twenty-one (24\%) of the comments focused on the student's desire to perform more direct patient skills (e.g., medication administration). The value of the experience was the most common theme within the comment questions, with $5 / 65(8 \%)$ finding limitations to the experience for learning, and 25/65 (38\%) enjoying the clinical experience, the unit or the clinical oversight.

\section{Discussion}

The COVID-19 pandemic has resulted in new challenges to student education, specifically to the clinical education 
of pre-licensure students. The authors found that using a novel approach to clinical education by introducing the intentional quality rounding process with pre-licensure student nurse training was a valuable experience. The summative evaluation process used by the authors revealed that the most critical elements associated with this type of clinical experience are organization, clear communication and defined guidelines for student skill development.

The use of intentional quality rounding as a direct patient care clinical activity was new for faculty and students. With a limited amount of time (less than 7 days) to prepare faculty and students for performing (students) and assessing (faculty) the rounding process, it was difficult to provide training for intentional quality rounding and the use of the checklist. Students pre-learning activities were limited by facility closures and requirements for social distancing. The pre-licensure students would typically have simulation experience, and time in the SON laboratory to prepare for the clinical experience. During the pandemic, the SON facilities were closed, and students learning was limited to video learning and webinars. The students were unable to practice the intentional quality rounding process until the day of the clinical experience, resulting in the students feeling less prepared for the experience.

The authors note a limitation for the activity related to evaluation. The activity was created as a quality improvement educational intervention. Quality improvement activities are rapid-cycle, iterative projects. There was no time for longitudinal evaluation of the activity, nor was there any opportunity to evaluate patient outcomes associated with quality rounds. Subjective student feedback was the only form of evaluation, given the rapid need for the project during COVID-19. However, this limitation of more rigorous assessment may provide the impetus for our future educational research utilizing the quality rounding process as a method for clinical nursing student education.

The logistics for organizing the clinical experience were challenging for the faculty, staff, and students. Social distancing and personal protective equipment requirements added a layer of complexity. Students and faculty met online, but the first face time with students and faculty was on the day of the clinical experience. Students were required to bring their own masks to the SON meeting site, but the leadership team for the hospital and SON provided a new mask for use on the days of clinical experience to ensure that the item was clean for use. The provision of a mask for each student and faculty required organization by the SON staff to order supplies, then meet the students for distribution.

The faculty and staff were challenged to provide enough space to effectively social distance given the number of students and faculty onsite at one time. The SON plaza is an outdoor location with large square footage, but this location still proved to be a challenge for organizing the student groups with enough physical distance. In addition, students wanted to engage in social conversations with peers and friends, making distancing and masking difficult to enforce.

The pre-licensure student evaluations of the clinical experience were overall positive. The students did have higher expectations for skill development than the clinical experience allowed. This was reflected in the two lowest evaluation ratings related to skill development and enjoying the experience. Pre-licensure students, particularly early-mid in program progression seemed focused on skill development. The clinical experience was specifically designed to limit student performance of skills due to the decreased number of available SON clinical faculty, and to ensure greater safety for the students during the pandemic.

The authors found it difficult to gauge the students' understanding of the ramifications of the rounding process from a broader perspective. The students focus on skill acquisition and direct patient care was reflected in the evaluation process with limited feedback by the students on the connection of patient care to safe, quality care and outcomes. The authors believe that it will be necessary to provide additional content specific to aspects of quality and safety that impact the patient and the organization. For example, the authors may need to incorporate training specific to external quality and safety reporting, with specific focus on measures that are relevant to falls, pressure injuries, restraints, and infections.

\section{Conclusions}

Nursing care is a primary driver for quality and safety in the hospital environment, and as such, we must ensure that our nursing educational processes teach the underpinnings associated with evidence-based quality and safety practices. During the COVID-19 pandemic, leaders have faced numerous challenges with ensuring that nursing student clinical education continues, particularly with limitations to direct patient care interactions. Developing clinical educational experiences that are innovative can be particularly challenging during a pandemic. However, one organizations use of intentional quality rounding as a mechanism for obtaining direct patient care hours during COVID-19 is an example of innovation during crisis that challenged nursing students to actively evaluate quality and safety in practice. While this project evolved out of necessity, future efforts to address new theoretical constructs such as quality and safety and care transitions we now consider central to the domain of nursing should be evaluated more extensively with research 
on processes and outcomes.

\section{CONFliCtS OF INTEREST Disclosure}

The authors declare that there is no conflict of interest.

\section{REFERENCES}

[1] Dang D, Dearholt S. Johns hopkins nursing evidence-based practice: model and guidelines. Third edition. Sigma Theta Tau International; 2018.

[2] McNeill L. Back to basics - how evidence-based nursing practice can prevent catheter-associated urinary tract infections. Urologic Nursing 2017 Jul/Aug. https : //doi .org/10.7257/1053-816X . 2017.3 7.4.204

[3] Melnyk B, Fineout-Overholt E. Evidence-based practice in nursing $\&$ healthcare: a guide to best practice. Fourth edition. WOLTERS KLUWER; 2019.

[4] Ea E, Alfes C. Innovative strategies in teaching nursing: exemplars of optimal learning outcomes. SPRINGER PUBLISHING CORP, LLC; 2021.

[5] Benner P. From novice to expert. The American Journal of Nursing. 1982 March; 82(3): 402-407. https : //doi : 10 . 2307/3462928

[6] Centers for Disease Control and Prevention. (n.d.). Coronavirus (COVID-19). Available from: https://www.cdc.gov/coronavi rus/2019-ncov/index.html

[7] Stevenson G. Students as active learners and teaching partners in the clinical setting. Nurse Educator. 2014 March/April; 39(2): 52-53. PMid:24535177 https://doi.org/10.1097/NNE.0000000000 000016

[8] Shin H, Sok S, Hyun K, et al. Competency and an active learning program in undergraduate nursing education. Journal of Advanced Nursing. 2015 March; 71(3): 591-598. PMid:25384709 https://doi.org/10.1111/jan.12564

[9] Groves P, Manges K. Understanding nursing handoffs: safety scholarship in nursing. Western Journal of Nursing Research. 2017 November; 39(11): 1391-1393. PMid:28835189 https ://doi .org/10.1 $177 / 0193945917727237$

[10] Wilkinson J, Treas L, Barnett K, et al. Procedure checklists for fundamentals of nursing. Third Edition. F.A. DAVIS; 2015.
[11] Program performance and evaluation office. Centers for Disease Control and Prevention; n.d [cited 2021 Dec 2]. Available from: https : //www.cdc.gov/eval/approach/

[12] Commission on collegiate nursing education. American Association of Colleges of Nursing; n.d. [cited 2021 Dec 2]. Available from: http://ccneaccreditation.org/

[13] Find the best nursing schools. U.S. News \& World Report; n.d [cited 2021 Dec 2]. Available from: https : //www . usnews. com/best-g raduate-schools/top-nursing-schools

[14] Magnet Recognition Program®). Nursingworld.Org; n.d [cited 2021 Dec 2]. Available from: https://www.nursingworld.org/org anizational-programs/magnet/

[15] What is NDNQI? (n.d.). Weebly.Com; n.d [cited 2021 Dec 2]. Available from: https://nursingandndnqi.weebly.com/wha t-is-ndnqi.html

[16] National Database of Nursing Quality Indicators (NDNQI); 2011 [cited 2021 Dec 2]. Available from: https ://nursingandndnqi . weebly.com/what-is-ndnqi.html

[17] Mcleod S. Likert scale definition, examples and analysis; 2020 [cited 2021 Dec 2]. Available from: https://www.simplypsychology .org/likert-scale.html

[18] Microsoft excel. Microsoft Office; n.d. [cited 2021 Dec 2]. Available from: https://www.microsoft.com/en-us/microsoft-365/excel

[19] Descriptive statistics: Definition \& charts and graphs. Statisticshowto.Com; 2018 [cited 2021 Dec 2]. Available from: https://www.statisticshowto.com/probability-and-s tatistics/descriptive-statistics/

[20] Knapp H. Introductory statistics using SPSS. Second Edition. SAGE; 2017.

[21] Statistical Package for the Social Sciences. IBM SPSS Statistics for Windows, Version 25.0. Armonk, NY: IBM CORP; 2017 [cited 2021 Dec 2]. Available from: https://www.ibm.com/products/sps s- 